\title{
Dynamic properties of arrays of ferromagnetic rectangular bars
}

Roman Adam, Yuri Khivintsev, Riccardo Hertel, Claus M. Schneider, Andrew Hutchison, Robert Camley, and Zbigniew Celinski

Citation: Journal of Applied Physics 101, 09F516 (2007);

View online: https://doi.org/10.1063/1.2713707

View Table of Contents: http://aip.scitation.org/toc/jap/101/9

Published by the American Institute of Physics

\section{Articles you may be interested in}

Spin wave resonance excitation in ferromagnetic films using planar waveguide structures Journal of Applied Physics 108, 023907 (2010); 10.1063/1.3435318

Annealing of $\mathrm{Ni}_{80} \mathrm{Fe}_{20} / \mathrm{Cu}$ and $\mathrm{Co} / \mathrm{Cu}$ multilayers

Journal of Applied Physics 87, 4852 (2000); 10.1063/1.373180

The design and verification of MuMax3

AIP Advances 4, 107133 (2014); 10.1063/1.4899186

Surface analysis of the Heusler Ni49.7 $\mathrm{Mn}_{29.1} \mathrm{Ga}_{21.2}$ Alloy: The composition, phase transition, and twinned microstructure of martensite

Journal of Applied Physics 120, 113905 (2016); 10.1063/1.4962648

Magnetostatic wave propagation in a periodic structure

Applied Physics Letters 29, 388 (2008); 10.1063/1.89098

Spin-dependent transport in multiwalled carbon nanotubes

Journal of Applied Physics 91, 7026 (2002); 10.1063/1.1452227

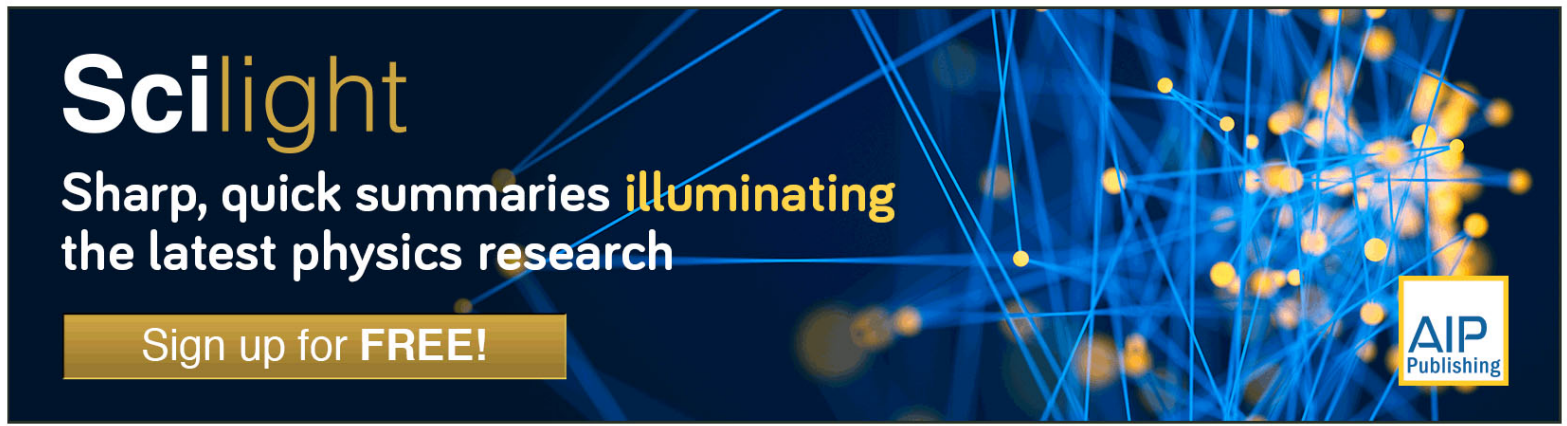




\title{
Dynamic properties of arrays of ferromagnetic rectangular bars
}

\author{
Roman Adam ${ }^{\text {a) }}$ \\ Institute of Solid State Research, Research Center Jülich, D-52425 Jülich, Germany \\ Yuri Khivintsev ${ }^{\text {b) }}$ \\ Center for Magnetism and Magnetic Nanostructures, University of Colorado at Colorado Springs, Colorado \\ Springs, Colorado 80918 \\ Riccardo Hertel and Claus M. Schneider \\ Institute of Solid State Research, Research Center Jülich, D-52425 Jülich, Germany

\begin{abstract}
Andrew Hutchison, Robert Camley, and Zbigniew Celinski
Center for Magnetism and Magnetic Nanostructures, University of Colorado at Colorado Springs, Colorado Springs, Colorado 80918
\end{abstract}

(Presented on 9 January 2007; received 31 October 2006; accepted 19 January 2007; published online 4 May 2007)

\begin{abstract}
Dynamic properties of arrays of rectangular bars were studied employing network analyzer ferromagnetic resonance and time-resolved magneto-optic Kerr effect (MOKE) measurements. The bars were patterned on top of coplanar waveguides and oriented with their long axis either parallel or perpendicular to the external magnetic field. Orientation of the bars parallel to the field results in an increase in the resonant frequency by up to $\sim 2 \mathrm{GHz}$, as well as the linewidth broadening by $0.3-1 \mathrm{GHz}$. The shift is shown to be in agreement with results expected from the difference of demagnetizing factors. The general behavior of the linewidth corresponds to calculations including shape anisotropy; however, the experimentally measured effect is much stronger than expected from theory. Fourier transforms of the time-resolved MOKE signal reveal the presence of several oscillatory modes, associated with the dynamics in the central part and at the borders of ferromagnetic elements. () 2007 American Institute of Physics. [DOI: 10.1063/1.2713707]
\end{abstract}

Possible future applications of magnetic thin films include high-frequency devices for communications and computing. ${ }^{1-3}$ To compete with existing applications, the magnetic devices must show superior performance in the gigahertz regime. The design and performance optimization of such devices require a detailed understanding of the underlying magnetization dynamics. Magnetostatic modes and spin waves in thin magnetic films attracted recently strong attention due to their importance for magnetic device operation. ${ }^{4-9}$ Patterned films and devices were shown to exhibit multiple magnetostatic or spin wave modes depending on their dimensions and the geometry. In our work we present the frequency- and the time-domain measurements of the magnetization dynamics in patterned Permalloy (Py) and cobalt (Co) films subjected to the continuous microwave pumping or to the short-pulse excitation.

Our structures were fabricated on top of GaAs substrates covered with a $1.5 \mu \mathrm{m}$ thick film of low temperature GaAs (LT-GaAs), grown at $\sim 250{ }^{\circ} \mathrm{C}$ using molecular beam epitaxy. After the LT-GaAs film growth, $50 \Omega$ coplanar waveguide (CPW) transmission lines were patterned by the deposition of a $200 \mathrm{~nm}$ thick Au film and lift-off. Transmission lines designed for the time-resolved measurements were terminated by a metal-semiconductor-metal photoconducting switch. Structures with 1:2 and 2:1 aspect ratios and dimen-

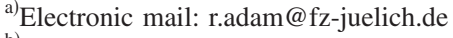

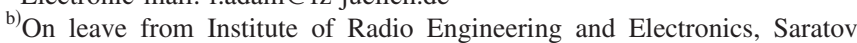
Department, 38 Zelenaya St., Saratov 410019, Russia.
}

sions ranging from $4 \times 8$ to $75 \times 150 \mu \mathrm{m}^{2}$ were subsequently patterned on top of the central CPW line using e-beam evaporation or magnetron sputtering and lift-off of Co or Py thin films.

To understand the high-frequency behavior of the small bars, we used network analyzer ferromagnetic resonance (NA-FMR) and time-resolved magneto-optic Kerr effect (TR-MOKE) measurements. In both experiments the static magnetic field was aligned along the CPW lines. The FMR measurements employed a continuous microwave driving field. The CPW transmission as a function of frequency was measured at different values of the static magnetic field. Further, the ferromagnetic resonance absorption was extracted by taking the transmission, $S_{12}$, at the desired field and subtracting an $S_{12}$ background signal at a different field.

In TR-MOKE experiments ferromagnetic structures were subjected to a train of magnetic pulses with the field oriented perpendicular to the CPW. Pulses were generated by the LT-GaAs photoconductive switch illuminated by $120 \mathrm{fs}$ short laser pulses from the Ti:sapphire laser. The synchronized low-power probe beam generated by the same laser was linearly polarized in the plane of incidence and then reflected from the cobalt surface under the incidence angle of $30^{\circ}$. The polarization state of the reflected probe beam corresponds to the instantaneous average magnetization of the array. The temporal evolution of the magnetization can be acquired by controlling the time delay between the excitation and the probe pulses. 

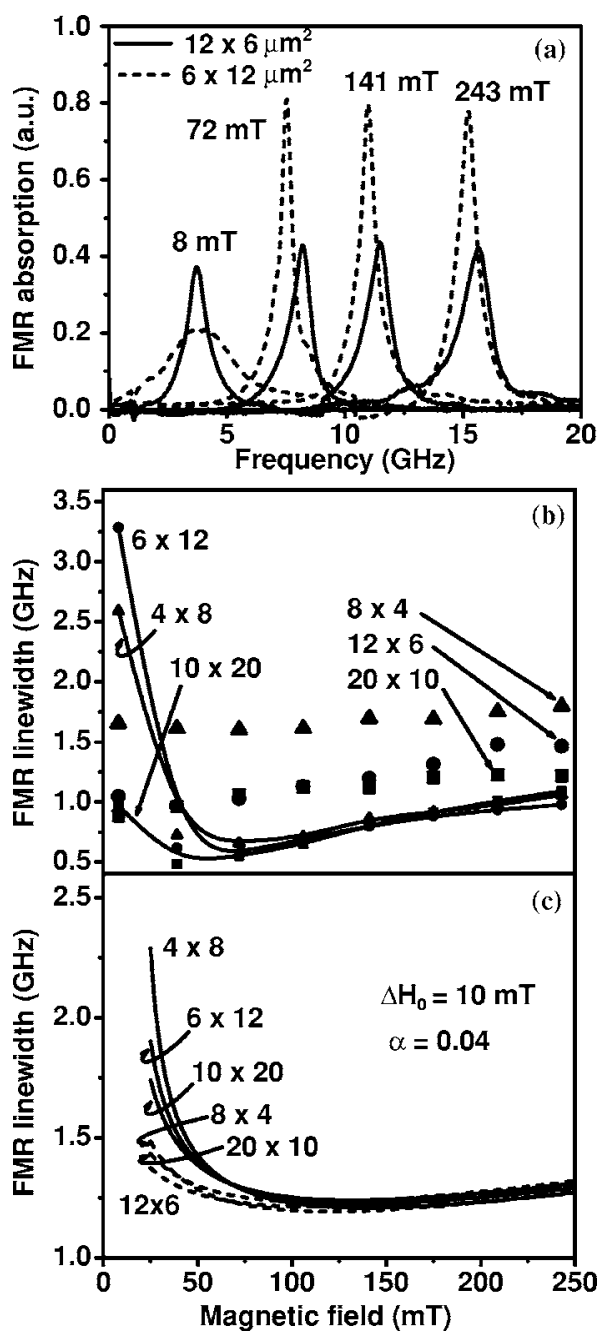

FIG. 1. FMR measurements on arrays of Py bars. (a) Resonance frequency as a function of field. $12 \times 6 \mu \mathrm{m}^{2}$ indicates a bar with long axis $(12 \mu \mathrm{m})$ parallel to the static magnetic field. (b) Field dependence of linewidth from experiment and (c) field dependence of linewidth from theory.

Figure 1(a) shows FMR measurements on $100 \mathrm{~nm}$ thick Py bars oriented with their long axis parallel or perpendicular to the static magnetic field. Changing the bar orientation from perpendicular to parallel shifts the resonance frequency up by about 1-2 GHz. The observed shift is in reasonable agreement with calculations of the ferromagnetic resonance frequency in external static field $H_{0}$ obtained from the formula $^{10}$

$$
\omega^{2}=\gamma^{2}\left[H_{0}+\left(N_{y}-N_{z}\right) 4 \pi M_{S}\right]\left[H_{0}+\left(N_{x}-N_{z}\right) 4 \pi M_{S}\right],
$$

taking into account the difference of demagnetizing factors $N$ for the two alignments and assuming a synchronized response of noninteracting ferromagnetic bars. In the formula, $\omega$ is the resonance frequency $\gamma$ is the gyromagnetic ratio, and $M_{S}$ is the saturation magnetization. The linewidths for bars with the two different orientations differ significantly. The general behavior of linewidth as a function of magnetic field corresponds reasonably to calculations ${ }^{11}$ including shape anisotropy, as is seen in Figs. 1(b) and 1(c). The bars with the long axis perpendicular to the static field have large linewidths at low field values and the linewidth rapidly decreases with increasing field. The bars with long axis parallel to the

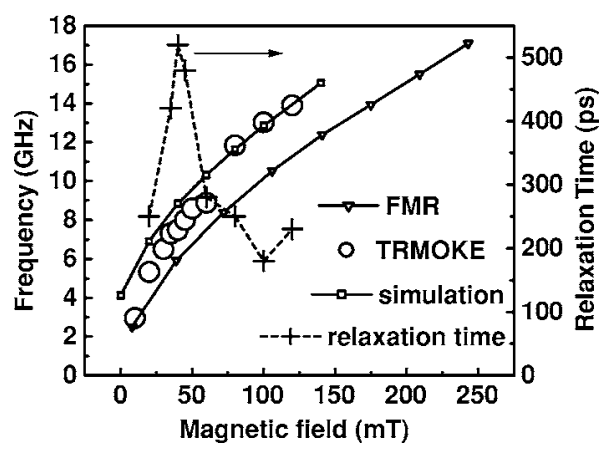

FIG. 2. Magnetic field dependence of the resonance frequency of Co bars ( $20 \times 10 \mu \mathrm{m}^{2}, 20 \mathrm{~nm}$ thick) obtained from FMR, and TR-MOKE measurements, and micromagnetic simulations. Crosses show the field dependence of $1 / e$ relaxation time from TR-MOKE.

applied field have a much flatter response. The theory and experiment are in general agreement, except that the experimental linewidth for the bars with long axis parallel to the applied field is much larger than is expected from the theory. It is not clear whether this is due to growth or patterning or due to some effect which is not included in the calculations.

Time-resolved measurements show several additional features not observed in FMR (see Figs. 2 and 3). Oscillatory TR-MOKE signals for different magnetic fields are shown in Figs. 3(a)-3(c) for a $20 \mathrm{~nm}$ thick Co bar aligned along the static field, with lateral dimensions $\left(50 \times 100 \mu \mathrm{m}^{2}\right)$ comparable to the probe-beam diameter $(\sim 50 \mu \mathrm{m})$. After a pulse excitation, the Kerr signal amplitude shows an initial increase during the first few oscillation cycles followed by a field-dependent exponential decrease. The gradual increase of the oscillation amplitude may be associated with the excitation and interference of multiple modes inside the sampled region. The decaying signal envelope is the measure of the system relaxation time. It shows strongly nonmonotonic dependence on the static field (see Fig. 2), suggesting a more complex response than the one observed in FMR.

To extract the oscillation power spectra we applied a Fourier transform (FT) to the time-resolved Kerr signal. Figures 3(d)-3(f) show the spectra corresponding to the timeresolved traces displayed in Figs. 3(a)-3(c). Compared to FMR, the spectra show not one but several pronounced oscillatory modes. Similar to the FMR, the frequency of the main oscillatory mode increases with the static magnetic field strength (see Fig. 2). In addition, and contrary to the FMR measurements, the power spectrum maxima show a strong variation with varying magnetic field. We ascribe the latter to the energy transfer from the edge-mode to the center-mode oscillations by increasing static magnetic field. Similar mode conversion has been observed earlier in rectangular Py platelets. ${ }^{12}$

To explain the TR-MOKE data we simulated the magnetization dynamics in a thin rectangular cobalt element subjected to $\sim 10 \mathrm{ps}$ magnetic pulse. The simulations were performed using a finite-element micromagnetic algorithm ${ }^{14}$ based on the Gilbert equation 

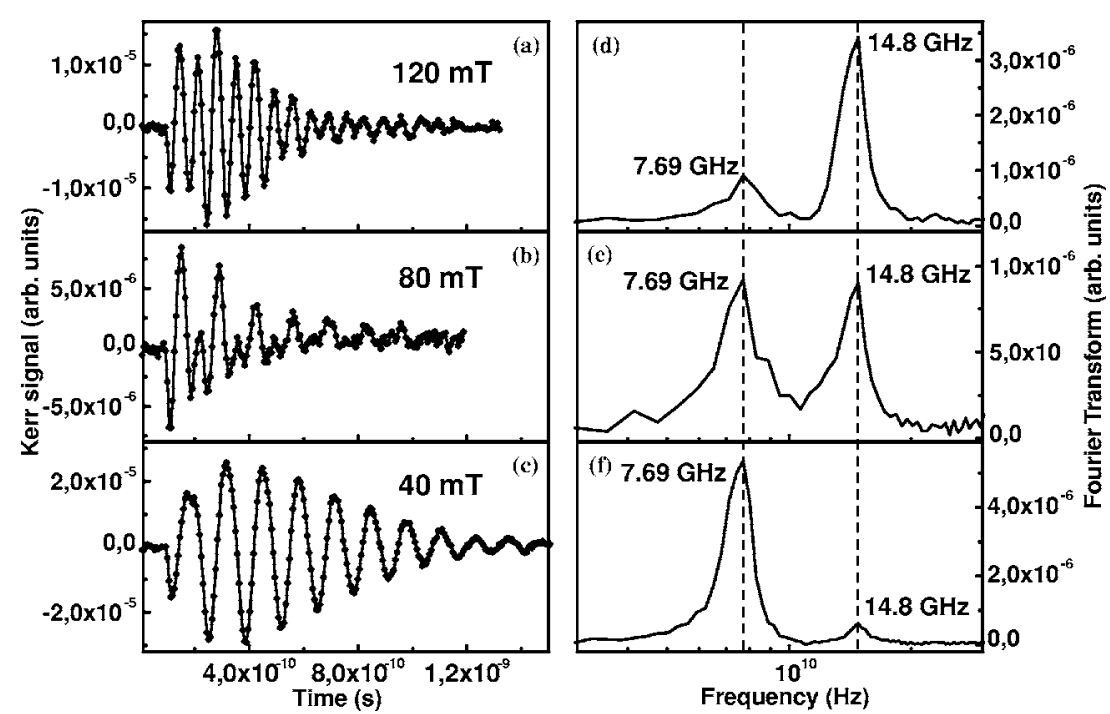

FIG. 3. Experimentally measured TRMOKE signal corresponding to 40, 80, and $120 \mathrm{mT}$ static magnetic fields (a)(c), and Fourier transforms of corresponding time-resolved traces (d)-(f).

$$
\frac{\partial \vec{M}}{\partial t}=-\gamma\left(\vec{M} \times \vec{H}_{\mathrm{eff}}\right)+\frac{\alpha}{M_{S}}\left(\vec{M} \times \frac{\partial \vec{M}}{\partial t}\right)
$$

that describes the dynamics of the magnetization vector $\boldsymbol{M}$ in an effective field $\boldsymbol{H}_{\text {eff. }}{ }^{13}$ The parameter $\alpha$ is the Gilbert damping constant. We first calculated the initial magnetic state of a $400 \times 200 \mathrm{~nm}^{2}$ Co bar placed in a static magnetic field aligned parallel to the long axis of the bar, and then applied the Gaussian-shaped 10 ps [full width at half maximum (FWHM)] magnetic pulse perpendicular to the static field direction. In all simulations we used $\alpha=0.02$ and mesh size $\leqslant 5 \mathrm{~nm}$. The inset of Fig. 4 shows the time evolution of the average out-of-plane magnetization component $\left\langle m_{z}(t)\right\rangle$ of the bar following the pulse excitation. Similar to TR-MOKE measurements, the power spectrum of the calculated response shown in Fig. 4 displays several distinct peaks. We

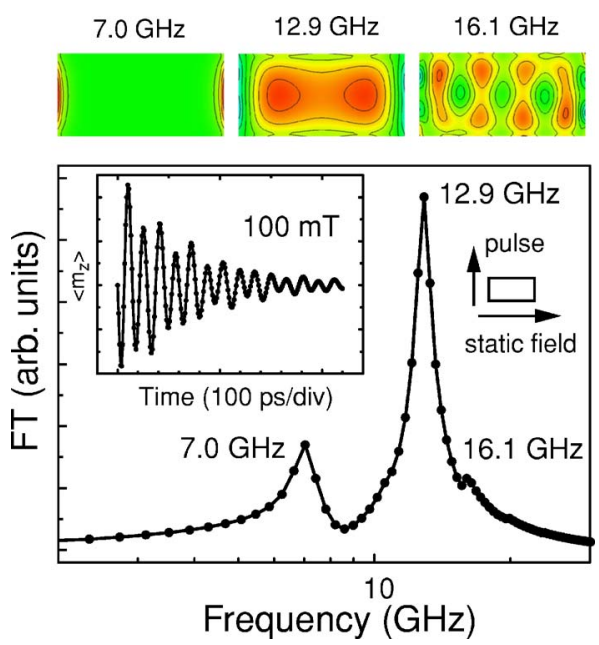

FIG. 4. (Color online) Fourier transform of the calculated temporal evolution of the volume-averaged magnetization component $\left\langle m_{z}\right\rangle$ at $100 \mathrm{mT}$ static field and $10 \mathrm{ps}$ pulse excitation. Inset shows the $\left\langle m_{z}(t)\right\rangle$ from micromagnetic simulations. Oscillatory modes shown for the marked frequencies correspond to the power spectrum maxima and were calculated by inverse Fourier analysis. used Fourier analysis to identify the modes corresponding to the power spectrum maxima. The simulations show that the main high- and low-frequency maxima are due to the oscillatory modes at the center and at the borders of the bar, respectively. Snapshots corresponding to the oscillatory modes are shown in Fig. 4.

In conclusion, we performed FMR and TR-MOKE experiments to test the magnetization dynamics in Permalloy and cobalt ferromagnetic arrays in continuous and pulse excitation regimes. The frequency shift observed in FMR with changes in orientation of the bars is consistent with the shift due to the demagnetization factors and the linewidth behavior can be accounted for by employing the calculations including shape anisotropy. Time-resolved measurements show a frequency behavior similar to that observed by FMR. In addition, however, multiple oscillatory modes have been observed in TR-MOKE possibly due to individual modes within the bar. Apparent energy transfer between modes has been observed by varying a static magnetic field. We employed micromagnetic simulations to reproduce main features of the TR-MOKE signal and to identify the oscillatory modes in the pulse excitation regime.

This work was supported by DOD (Grant No. W911NF04-1-0247).

${ }^{1}$ M. Yamaguchi et al., J. Magn. Magn. Mater. 304, 208 (2006).

${ }^{2}$ Y. Zhou et al., IEEE Trans. Magn. 42, 2425 (2006).

${ }^{3}$ I. N. Krivorotov et al., Science 307, 228 (2005).

${ }^{4}$ M. Yan et al., Phys. Rev. B 73, 014425 (2006).

${ }^{5}$ R. D. McMichael et al., J. Appl. Phys. 97, 10J901 (2005).

${ }^{6}$ J. P. Park et al., Phys. Rev. B 67, 020403 (2003).

${ }^{7}$ G. Gubbiotti et al., J. Phys.: Condens. Matter 16, 7709 (2004).

${ }^{8}$ C. Bayer et al., Phys. Rev. B 72, 064427 (2005).

${ }^{9}$ R. D. McMichael et al., J. Appl. Phys. 89, 7603 (2001).

${ }^{10}$ Ch. Kittel, Phys. Rev. 73, 155 (1948).

${ }^{11}$ B. Kuanr et al., Appl. Phys. Lett. 87, 012502 (2005).

${ }^{12}$ M. Buess et al., Phys. Rev. Lett. 94, 127205 (2005).

${ }^{13}$ W. F. Brown, Jr., Micromagnetics (Wiley Interscience, New York, 1963).

${ }^{14}$ R. Hertel et al., Phys. Rev. Lett. 93, 257202 (2004). 